\title{
Moving Horizon Model Based Control in the Presence of Feedback Noise
}

\author{
Liu Zhimin \\ School of Mechanical \\ Engineering, Beijing Institute \\ of Petrochemical Technology \\ Beijing, China
}

\author{
Wang Joe Keqi \\ Department of Mechanical \\ Engineering, \\ University of Thessaly, \\ Karditsa, Greece
}

\author{
Arthur, $\mathrm{Hu}$ \\ Department of Electrical and \\ Electronics Engineering, \\ Kagoshima University, \\ Japan
}

\begin{abstract}
This paper studies the performance of networked control systems with a receding horizon controller. It is also assumed that there exists exogenous noise signal in feedback channel, modeled as a stochastic process. The impact of this noise on the closed-loop system performance is examined through both theoretical analysis and numerical experiments. An adaptive compensator is proposed to assist the original receding horizon controller. The performance of this solution is verified through simulation.
\end{abstract}

Keywords: Model predictive control, networked control system, noise, tracking

\section{INTRODUCTION}

With the advance of communication technology, the concept of networked control system is becoming more and more popular both in academia and industry. When a traditional feedback control system is closed with a communication channel (for example, wireless network), which may be shared with other nodes besides the control system, then the control system is termed as a networked control system (NCS). A typical networked control system has four basic components: sensors, controllers, actuators and communication channel. Networked control system has many advantages over traditional control systems, for examples, it enabled the execution of several tasks from long distance; the complexity and maintenance cost of the system is potentially lower because of elimination of unnecessary wiring; upgrading or modifying components becomes relatively easy and low-cost. However, these advantages do not come for free. There are also technical challenges that come with the networked control system, which gave rise to important research topics.

It is well known that when sensors and actuators communicate with a remote controller over a possibly noisy communication network, estimation as well as controller design becomes challenging [1]. For example, stability of the closed loop requires additional conditions on the communication channel used. In $[3,4,5,8]$, it is pointed out that the capacity of communication channel in the feedback loop should be lower-bounded to guarantee closed-loop stability. $[6,9,10,13,14]$ studied the performance limitation of control system and related it to the information content in the feedback channel. Various coding schemes for networked control are studied under different channel assumptions [2, 11]. Event-triggered control is also proposed [7, 12, 15, 16, 17] which can save transmission bandwidth while maintaining closed-loop stability.

In this paper we are interested in the performance of a networked receding horizon controller, also known as model predictive control. It is assumed that there exists exogenous noise signal in feedback channel which corrupts the measurement. In this study package drop, delay and quantization effects are ignored to avoid unnecessary complexity. This setup is very realistic since model predictive control is widely used in industrial applications, noisy signals in communication network is also quite common. We studied this scenario with both theoretical analysis and numerical experiment, an adaptive compensator is also proposed to improve the performance of the original receding horizon controller.

The rest part of the paper is organized as follows: in section 2 the problem formulation is presented. Section 3 describes the modified MPC algorithm with adaptive compensator, experiment results are in section 4 while section 5 summarizes this study.

\section{PROBLEM FORMULATION}

\subsection{Model predictive control over communication channel}

Model predictive control refers to a class of control algorithms that rely on an explicit plant model to predict its future response. At each control interval an MPC algorithm attempts to optimize future plant behavior by computing a sequence of future control variable adjustments. The first input in the optimal sequence is then sent to the actuators, and the entire calculation is repeated at the next time intervals. Originally developed to meet the specialized control needs of power plants and petroleum refineries, MPC technology are now very popular in a wide variety of application areas including chemicals, automotive, and aerospace applications $[18,19,20]$.

Figure.1 shows a closed-loop system with model predictive control and noisy feedback channel over the network. We consider the case when the plant is linear, the equation that describe the system dynamics is:

$$
x(t+1)=A x(t)+B u(t)
$$

Assume that the noise that corrupts the state measurement is i.i.d. Gaussian, denoted as $w(t)$, the model predictive control problem can be formulated as

$$
\begin{gathered}
\min _{u} \sum_{k=t}^{t+N} J(x(k), u(k)) \\
g_{i}(x(k), u(k)) \geq 0, i=1,2 \ldots m \\
x(k+1)=A x(k)+B u(k), k=t+1, \ldots t+N-1, \\
x(t)=x^{*}
\end{gathered}
$$


Here $x^{*}$ is the noisy state measurement at time $t$.

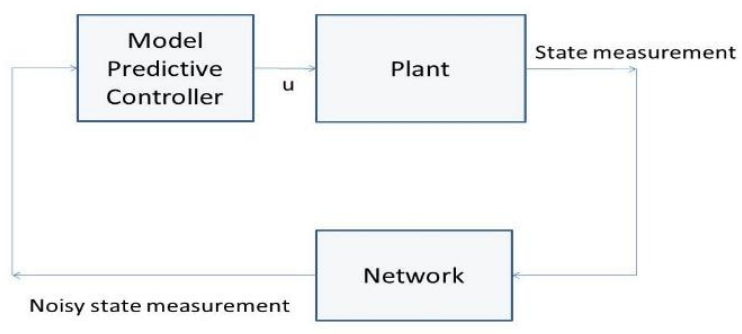

Figure.1 Model predictive control over noisy communication network.

\subsection{Effect of exogenous noise on performance}

The noise in the feedback channel has a negative impact on the performance of closed-loop system. With the noise $w(t)$, the initial state $x^{*}$ at time $t$ is no longer accurate. Optimization based on this inaccurate initial state may lead to sub-optimal control sequences in the following time steps. This suboptimal control gives rise to deterioration of closed-loop performance.

\section{MODIFIED MPC ALGORITHM}

In this section we propose a modification of the original model predictive control framework. This is achieved by adding an adaptive compensator to the original design, as depicted in Figure 2. Note that there is no noisy feedback channel in this figure. This compensator is actually an adaptive controller that helps the model predictive controller by adaptively correcting the state error in the measurement.

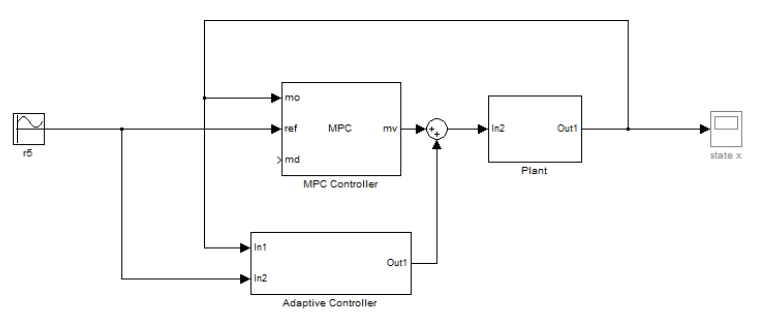

Figure. 2 Modified model predictive control with adaptive compensator

The adaptive controller takes both the reference signal and measured noisy state as input, with a high-gain adaptive law, it can track the state changes and generate a proper control action that adds to the control action of the MPC controller to compensate the effect of measurement noise. In the next section we use experiments to show the effectiveness of the proposed approach.

Some theoretical analysis needed.

\section{SIMULATION}

In this section we use a simple example to illustrate the modified model predictive control approach we proposed. For a linear time invariant dynamical system with transfer function

$$
G(s)=\frac{458}{s^{3}+31 s^{2}+259 s+229}
$$

we consider a tracking task where the objective is to make the output of the dynamical system track a given sinusoidal reference signal $r(t)=\sin \left(\frac{\pi}{5} t\right)$. The output $y(t)$ is also measured and sent to a model predictive controller through noise-corrupted communication channel. The noise is modeled as i.i.d. Gaussian with zero mean and variance value 5 .

The model predictive controller has a sampling period of 0.1 second. We chose a prediction horizon of 60 steps and a control horizon of 1 step. Quadratic functions are used as objective function of the resulting optimization problem:

$$
\begin{aligned}
& J(x, u)=\sum_{t=1}^{60}\left((y(t)-r(t))^{T} Q(y(t)-r(t))+\right. \\
& \left.u(t)^{T} R u(t)\right)
\end{aligned}
$$

Here $y(t)$ and $r(t)$ are the output and the sinusoidal reference signal, respectively. We implemented this control solution using MPC toolbox from MATLAB. Our proposed modified MPC is also implemented with a carefully tuned adaptive control block. Here we made the assumption that the initial condition of the controlled plant is known to the adaptive controller.

The performance of these two control solutions are compared in Figure.3. It can be observed that the output of modified MPC is much better.

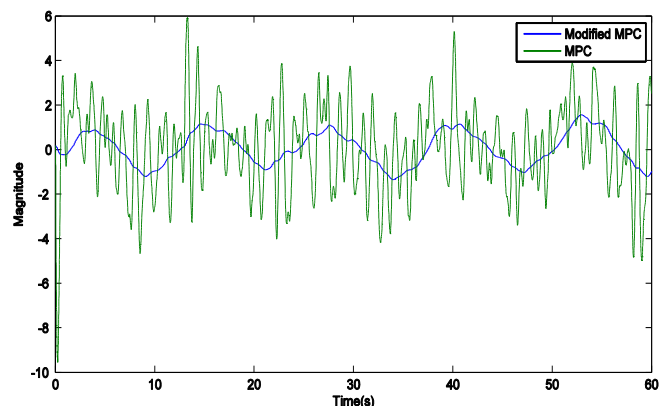

Figure 3. Comparing the tracking performance of traditional MPC and proposed modified MPC

We also test the two control solutions with a square wave reference input. With large noise in the feedback channel, regular MPC cannot track the steps very well. Using the proposed modified MPC the tracking performance is much better, as depicted in Figure 4 and Figure 5, respectively.

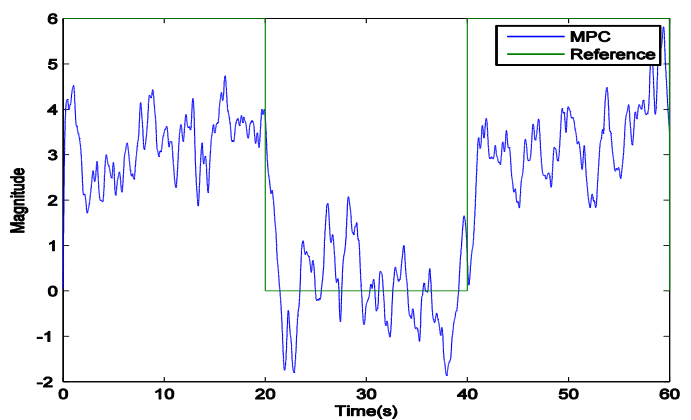

Figure 4.Tracking performance of regular MPC with square wave as reference input. 


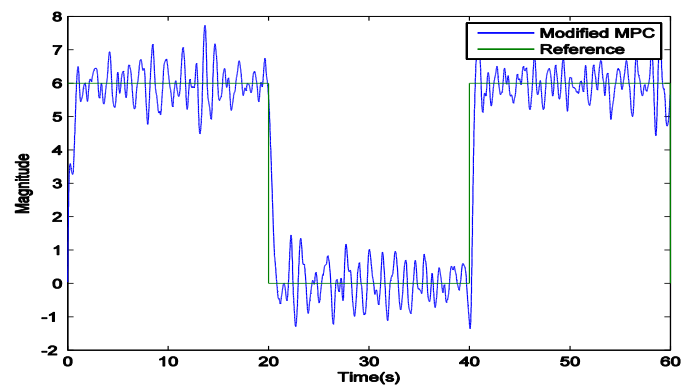

Figure 5.Tracking performance of proposed MPC with square wave as reference input.

\section{CONCLUSION}

In this paper we studied the receding horizon model based control (MPC) when the state measurements are obtained through noisy communication channel. A modified MPC controller is proposed which use an extra adaptive controller to compensate for the exogenous noise. Experiment results show that the proposed method outperforms regular MPC solution in typical output tracking application.

\section{REFERENCES:}

[1] Baillieul, John and Antsaklis, Panos J., "Control and Communication Challenges in Networked Real-Time Systems", Proceedings of the IEEE, 95(1) pp.9 - 28, 2007

[2] Gomez-Estern, F.; Canudas-de-Wit, Carlos; Rubio, F.R.; Fornes, J., "Adaptive Delta-modulation Coding for Networked Controlled Systems," American Control Conference, pp. 4911-4916, 2007

[3] Sekhar Tatikonda, S. Mitter, "Control Under Communication Constraints", IEEE Transactions on Automatic Control, 49, 1056-1068., 2004

[4] Sekhar Tatikonda, S. Mitter, "Control Over Noisy Channels", IEEE Transactions on Automatic Control, 49, 1196-1201, 2004,

[5] G. N. Nair \& R. J. Evans, "Stabilization with data-ratelimited feedback: tightest attainable bounds", Systems \& Control Letters, vol. 41, no. 1, pp. 49-56, 2000.

[6] Sun Yu, Prashant G. Mehta, "Fundamental performance limitations with Kullback-Leibler control cost," in Proceedings of Conference on Decision \& Control, pp.7063-7068, 2010.

[7] Tabuada, P., "Event-Triggered Real-Time Scheduling of Stabilizing Control Tasks", IEEE Transactions on Automatic Control, 52(9), pp.1680-1685, 2007

[8] Nicola Elia: Remote stabilization over fading channels. Systems \& Control Letters, vol.54, no.3, pp.237-249, 2005.

[9] Martins, Nuno C., Dahleh, Murither A., "Feedback Control in the Presence of Noisy Channels: 'Bode-Like' Fundamental Limitations of Performance", IEEE Transactions on Automatic Control, vol.53, issue.7, pp. $1604-1615,2008$
[10] Yu Sun and P. G. Mehta, "Fundamental performance limitations via entropy estimates with hidden Markov models," in Procs. of Conference on Decision \& Control, pp. 3982-3988, 2007.

[11] Canudas-de-Wit, Carlos; Rubio, F.R.; Fornes, J.; GomezEstern, F., "Differential coding in networked controlled linear systems," American Control Conference, pp. 1416, 2006

[12] X. Wang and M. Lemmon, Event-Triggering in Distributed Networked Control Systems, IEEE Transactions on Automatic Control, vol. 56, no. 3, 586601,2011

[13] G. Zang, and P. A. Iglesias, "Nonlinear extension of Bode's integral based on an information theoretic interpretation", Systems and Control Letters, vol.50, pp.11-29, 2003.

[14] Sun Yu, Prashant G. Mehta, "Bode-Like Fundamental Performance Limitations in Control of Nonlinear Systems". IEEE Transaction on Automatic Control, vol. 55, pp.1390-1405, 2010.

[15] Wang, Xiaofeng; Yu Sun; Hovakimyan, N., "Relaxing the consistency condition in distributed event-triggered networked control systems," IEEE Conference on Decision and Control (CDC) pp.4727-4732, 2010

[16] Dimarogonas, D.V.; Johansson, K.H., "Event-triggered control for multi-agent systems," IEEE Conference on Decision and Control, pp.7131-7136, 2009

[17] Xiaofeng Wang, Y. Sun, Naira Hovakimyan: Asynchronous task execution in networked control systems using decentralized event-triggering. Systems \& Control Letters 61(9), pp. 936-944, 2012

[18] Camponogara, E.; Jia, D.; Krogh, B.H.; Talukdar, S., "Distributed model predictive control," IEEE Control Systems Magazine, vol.22, no.1, pp.44-52, 2002

[19] Yang Wang; Boyd, S., "Fast Model Predictive Control Using Online Optimization," Control Systems Technology, IEEE Transactions on, vol.18, no.2, pp.267,278, March 2010

[20] Rawlings, J.B., "Tutorial overview of model predictive control," IEEE Control Systems Magazine, vol.20, no.3, pp.38-52, 2000 